\title{
Editorial ,Studienabbruch““
}

\section{Martin Neugebauer $[$ - Ulrich Heublein • Bettina Hannover}

Fast 3 Millionen! Niemals zuvor in der Geschichte haben so viele Menschen in Deutschland studiert wie im Wintersemester 2018/2019. Gut 50\% beträgt der Anteil einer Alterskohorte, der ein Hochschulstudium beginnt. Studieren, damit verbinden viele persönliche und intellektuelle Freiheit, nach dem Ende der Schulzeit, die oft als wenig selbstbestimmt erlebt wird. Doch nicht für jeden ist das Studium eine von Unbeschwertheit und Suche nach intellektuellen Herausforderungen geprägte Lebensphase. Oftmals geht dieser Lebensabschnitt auch mit Unsicherheit einher, mit Selbstzweifeln, Überforderung und Demotivation. Dies führt nicht selten zu einer Umorientierung weg von der Hochschule, verbunden mit einem Studienabbruch. Abbruch, das klingt negativ, und verkennt den Umstand, dass mit einer solchen Entscheidung für manche Betroffene eine gewinnbringende Phase der Neuorientierung beginnt. Doch auch wenn eine solche Neuorientierung im Einzelfall sinnvoll sein kann, gelten hohe Abbruchquoten auf gesellschaftlicher Ebene als problematisch. Denn volkswirtschaftlich betrachtet bedeuten sie hohe Investitionen in Personen, die schlussendlich dem Arbeitsmarkt nicht als akademisch ausgebildete Fachkräfte zur Verfügung stehen. Gut ausgebildete Akademikerinnen und Akademiker leisten aber, das ist weitgehend unbestritten, einen wesentlichen Beitrag für die Wettbewerbsfähigkeit und Innovationskraft eines Landes. Der kürzlich beschlossene ,Zukunfts-

Prof. Dr. M. Neugebauer $(\bowtie) \cdot$ Prof. Dr. B. Hannover

Freie Universität Berlin, Habelschwerdter Allee 45, 14195 Berlin, Deutschland

E-Mail: martin.neugebauer@fu-berlin.de

Prof. Dr. B. Hannover

E-Mail: bettina.hannover@fu-berlin.de

Dr. U. Heublein

Deutsches Zentrum für Hochschul- und Wissenschaftsforschung (DZHW), Lange Laube

12, 30159 Hannover, Deutschland

E-Mail: heublein@dzhw.eu 
vertrag Studium und Lehre stärken“, mit dem Milliarden an Fördermitteln an die Hochschulen verteilt werden, hat dies erneut betont.

Im Zusammenhang mit hochschulpolitischen Debatten zur Qualität des Hochschulstudiums und im Zuge vermehrter Bemühungen der deutschen Hochschulen zur Sicherung des Studienerfolgs rückt das Thema Studienabbruch auch wieder stärker in den Fokus der empirischen Bildungs- und Hochschulforschung. Besonders relevant ist das Thema in den naturwissenschaftlich-technischen Disziplinen, wo die Abbruchquoten überdurchschnittlich hoch sind und gleichzeitig die Nachfrage nach akademischen Fachkräften höher ist als das Angebot an Hochschulabsolventinnen und -absolventen.

Wie viele Menschen in Deutschland tatsächlich ein Studium abbrechen, wissen wir nicht. Das mag erstaunlich klingen, doch bislang liegen nur Schätzungen vor, die je nach Methode zu sehr unterschiedlichen Ergebnissen kommen. Bislang fehlen administrative Daten zu individuellen Studienverläufen auch über Hochschulstandorte hinweg, mit denen Abbruchquoten zuverlässig ermittelt werden könnten. Auch zu anderen zentralen Fragen der Studienabbruchforschung ist unser Wissen begrenzt. Zwar untersuchen etliche Studien die individuellen Ursachen eines Abbruchs, allerdings sind diese oft als Querschnittsuntersuchung angelegt oder auf einzelne Hochschulstandorte begrenzt. Es mangelt an überregionalen prospektiven Längsschnittstudien, mit denen sich kausale Wirkungszusammenhänge bis zur vorzeitigen Exmatrikulation nachvollziehen lassen. Zu Fragen nach den ökonomischen oder psychologischen Folgen von Studienabbrüchen liegen kaum Untersuchungen vor. Für eine angemessene Bewertung des Phänomens Studienabbruch ist dies jedoch von entscheidender Bedeutung. Schließlich besteht dringender Forschungsbedarf zur Frage, wie sich drohende Studienabbrüche frühzeitig erkennen und mit welchen Maßnahmen sie sich effektiv verhindern lassen.

Das Feld der Studienabbruchforschung ist in Bewegung, auf der Suche nach Antworten auf diese Fragen. Die Förderlinie ,Studienerfolg und Studienabbruch“ des Bundesministeriums für Bildung und Forschung (BMBF) finanziert seit 2017 insgesamt 19 (Verbund-)Projekte zum Thema. Auch außerhalb der BMBF-Förderlinie laufen derzeit anspruchsvolle Forschungsprojekte zum Themenbereich Studienabbruch. Hier knüpft dieses Themenheft an. Es systematisiert den aktuellen Forschungsstand und stellt ausgewählte empirische Befunde aus unterschiedlichen Forschungsvorhaben gebündelt dar. Dabei haben wir die Beiträge bewusst aus verschiedenen Disziplinen zusammengestellt, denn Hochschulforschung ist interdisziplinär. Weiter wurden die Beiträge so gewählt, dass sie ein breites Spektrum an Datengrundlagen und Methoden repräsentieren: Neben Sekundärdatenanalysen (z. B. NEPS) finden sich Studien mit experimentellen und längsschnittlichen Primärdatenerhebungen oder dem Einsatz von Methoden des maschinellen Lernens.

Zunächst fassen Martin Neugebauer, Ulrich Heublein und Annabell Daniel im Stichwortbeitrag den Stand der Forschung zu Studienabbrüchen in Deutschland zusammen. Sie gehen dabei auf die vier zentralen Fragen der Studienabbruchforschung ein, die hier bereits angeklungen sind: Wie viele Studierende brechen ihr Studium ab? Warum brechen sie ihr Studium ab? Was sind die Folgen eines Abbruchs? Wie kann Studienabbrüchen vorgebeugt werden? Dabei betonen die Autorinnen und Autoren die vielfältigen methodischen Herausforderungen der Studienabbruchfor- 
schung, die bei der Einordnung der Befunde zu berücksichtigen sind. Die Zusammenfassung des Forschungsstandes zeigt unter anderem, dass sich die Abbruchquoten in den vergangenen Jahren nur unwesentlich geändert haben. Angesichts der massiven Hochschulexpansion und der damit verbundenen wachsenden Heterogenität der Studierendenschaft wäre durchaus ein Anstieg zu erwarten gewesen. Dass dies nicht geschehen ist, mag auch daran liegen, dass die Hochschulen mit wirksamen Maßnahmen der Qualitätssicherung auf die gestiegene Studiennachfrage reagiert haben. Ein weiterer interessanter Befund lautet, dass bis zu $40 \%$ aller beobachteten Studienabbrüche sich später als Studienunterbrechungen erweisen. Ein vermeintlicher Abbruch stellt also keineswegs immer eine endgültige Entscheidung gegen ein Hochschulstudium dar.

Ursachen, Folgen, Präventionsmaßnahmen - in dieser Reihenfolge sind die nachfolgenden empirischen Beiträge im Heft angeordnet.

Der erste Beitrag von Sören Isleib, Andreas Woisch und Ulrich Heublein widmet sich der Frage, welche Faktoren fächer- und standortübergreifend den Prozess des Studienabbruchs beeinflussen. Die Autoren verfolgen das Ziel, einen breiten Kranz an theoretisch wirksamen Prädiktoren des Studienabbruchs simultan zu analysieren. In ihrer Re-Analyse der querschnittlichen DZHW-Exmatrikuliertenbefragung wird der bisherige Forschungsstand weitgehend bestätigt: Studienabbrecherinnen und -abbrecher haben schlechtere Abiturnoten als Studierende, die regulär abschließen, sie gelangen häufiger über nicht-traditionelle Wege zum Studium, sie bewerten die akademische und soziale Integration an den Hochschulen negativer, genauso wie die Studienbedingungen und die Höhe der Studienanforderungen. Absolventinnen und Absolventen waren hingegen häufiger studiennah erwerbstätig und berichteten häufiger von einer gesicherten Studienfinanzierung als Studierende, die sich vorzeitig exmatrikulierten.

Die nächsten beiden Beiträge widmen sich der Ursachensuche für Studienabbrüche in naturwissenschaftlich-technischen Studiengängen. Jens Fleischer, Detlev Leutner, Matthias Brand, Hans Fischer, Martin Lang, Philipp Schmiemann und Elke Sumfleth legen den Fokus auf die Fächer Biologie, Chemie, Bauingenieurwesen und Physik. In ihrem Beitrag, der auf längsschnittlichen Daten zur Studieneingangsphase an zwei Hochschulstandorten basiert, zeigt sich, dass kognitive Fähigkeiten den Studienabbruch ebenso vorhersagen wie motivationale und interessensbezogene Personenmerkmale. Die Zufriedenheit mit den Studieninhalten erweist sich dabei als Mediator, über den ein Teil der Effekte der anderen Prädiktoren auf die Abbruchsintention vermittelt wird. Die Abbruchsintention stellt einen frühen Indikator für einen späteren Studienabbruch dar. Die Ergebnisse zeigen sowohl Gemeinsamkeiten als auch Unterschiede zwischen den betrachteten Studiengängen hinsichtlich der Bedeutung der einzelnen Prädiktoren.

Elisabeth Höhne und Lysann Zander legen den Fokus auf das Fach Informatik. Vor dem Hintergrund der geringen Frauenquote in diesem Fach untersuchen sie, welchen Einfluss die Unsicherheit über die soziale Zugehörigkeit (,,belonging uncertainty“) zum Studienfach auf die Studienabbruchsintention hat. Auf Basis einer Erhebung mit Erstsemesterstudierenden einer deutschen Hochschule zeigt sich, dass zum einen weibliche Studierende eine größere Unsicherheit bezogen auf ihre soziale Zugehörigkeit erleben als männliche Studierende, dass aber zum anderen diese 
Zugehörigkeitsunsicherheit bei beiden Geschlechtern prädiktiv für die Studienabbruchsintention ist.

Kerstin Schneider, Johannes Berens und Julian Burghoff befassen sich in ihrem Beitrag mit Frühwarnsystemen zur Identifikation abbruchgefährdeter Studierender. Zunehmend werden solche Systeme an deutschen Hochschulen eingesetzt, denn sie ermöglichen es den Hochschulen, Hilfs- und Beratungsangebote frühzeitig und passgenau anzubieten - und so die betroffenen Studierenden bei Schwierigkeiten zu unterstützen. Allerdings ist der Einsatz von Frühwarnsystemen mit guter Prognosegenauigkeit oft aufwendig und komplex, was Hochschulen davon abhalten kann, sie zu nutzen. Der Beitrag prüft daher mittels Verfahren des maschinellen Lernens und anhand administrativer Studierendendaten, wie gut ein drohender Studienabbruch weitgehend automatisiert prognostiziert werden kann. Die Analysen zeigen, dass ein automatisiertes Prognosemodell auf Basis der Variablen, die an Hochschulen im Rahmen des Hochschulstatistikgesetzes ohnehin erhoben werden, eine hohe Prognosequalität aufweist und dass dieses Modell durch die eingesetzten Methoden des maschinellen Lernens an weiteren deutschen Hochschulen ohne größere Anpassung eingesetzt werden könnte. Es zeigt sich aber auch, dass eine Reduktion der Variablenanzahl im Sinne eines noch effizienteren Monitorings mit deutlichen Einschränkungen in der Prognosegüte einhergeht, zumindest für Studierende in frühen Semestern, in denen die meisten Abbrüche zu verzeichnen sind.

Da bislang kaum etwas zu der Situation von Abbrecherinnen und Abbrechern auf dem Arbeitsmarkt und damit zu den beruflichen Folgen einer vorzeitigen Exmatrikulation bekannt ist, untersucht der Beitrag von Annabell Daniel, Martin Neugebauer und Rainer Watermann die Einstellungschancen für diese Personengruppe. Der Beitrag fokussiert dabei auf Ausbildungsstellen, denn in Deutschland versuchen die meisten Studienabbrecherinnen und -abbrecher, über die Aufnahme einer beruflichen Ausbildung auf dem Arbeitsmarkt Fuß zu fassen. Im Rahmen eines faktoriellen Surveys bewerteten Arbeitgeberinnen und Arbeitgeber mehr als 5000 fiktive Bewerbungsprofile, in denen das Vorliegen eines Studienabbruchs neben weiteren Merkmalen experimentell variiert wurde. Im Ergebnis zeigt sich, dass Studienabbrecherinnen und -abbrecher im Durchschnitt mit höherer Wahrscheinlichkeit zu einem Vorstellungsgespräch eingeladen werden als Abiturientinnen und Abiturienten. Darüber hinaus begünstigen gute Studienleistungen, eine fachliche Nähe zwischen dem Studienfach und dem Ausbildungsberuf sowie ein berufsrelevantes Praktikum die Einladungswahrscheinlichkeit, während ein später Abbruchzeitpunkt diese verringert. Die Studie stellt praktisch bedeutsame Befunde zu den kausalen Folgen eines Studienabbruchs bereit, die die häufig diskutierte Annahme, Studienabbrecherinnen und -abbrechern hafte ein Makel des Scheiterns an, relativieren.

Der abschließende Beitrag von Nicole Tieben untersucht eine Maßnahme, die die Studienerfolgswahrscheinlichkeit erhöhen soll: Brückenkurse. Die Autorin fokussiert auf ingenieurswissenschaftliche Studiengänge, in denen Brückenkurse häufig mit dem Ziel angeboten werden, vermeintliche Defizite in der Studienvorbereitung zu kompensieren. Doch lassen sich dadurch Studienabbrüche verhindern? Der Beitrag verwendet Daten der Studierendenkohorte des Nationalen Bildungspanels. Durch Entropy Balancing wird versucht, Teilnehmende und Nichtteilnehmende auszubalancieren, um den Kausaleffekt der Brückenkursteilnahme besser isolieren zu 
können. Dabei finden sich Hinweise, dass Brückenkursteilnehmende an Universitäten seltener ein Studium abbrechen als Nichtteilnehmende, während an Fachhochschulen die Teilnahme an einem Brückenkurs in keinem Zusammenhang mit dem Studienabbruch steht. Weiterhin zeigt sich, dass die eigentlichen Adressaten von Brückenkursen, Studierende mit mathematischen Defiziten, nicht immer erreicht werden.

Insgesamt zeigen die Beiträge: Das interdisziplinäre Feld der Studienabbruchforschung in Deutschland ist in Bewegung! An vielen Stellen gelingt es, mit neuen Daten alte Fragen besser als zuvor zu beantworten. Deutlich wird aber auch, dass die Entwicklung von Forschungsdesigns, die kausale Aussagen zu Ursachen, Folgen und Maßnahmen erlauben, nach wie vor eine große Herausforderung darstellt. Hier sind weitere innovative Forschungsbemühungen erforderlich. 